\title{
Classification and Reconstruction From Random Projections for Hyperspectral Imagery
}

\author{
Wei Li, Student Member, IEEE, Saurabh Prasad, Member, IEEE, and James E. Fowler, Senior Member, IEEE
}

\begin{abstract}
There is increasing interest in dimensionality reduction through random projections due in part to the emerging paradigm of compressed sensing. It is anticipated that signal acquisition with random projections will decrease signal-sensing costs significantly; moreover, it has been demonstrated that both supervised and unsupervised statistical learning algorithms work reliably within randomly projected subspaces. Capitalizing on this latter development, several class-dependent strategies are proposed for the reconstruction of hyperspectral imagery from random projections. In this approach, each hyperspectral pixel is first classified into one of several pixel groups using either a conventional supervised classifier or an unsupervised clustering algorithm. After the grouping procedure, a suitable reconstruction method, such as compressive projection principal component analysis, is employed independently within each group. Experimental results confirm that such class-dependent reconstruction, which employs statistics pertinent to each class as opposed to the global statistics estimated over the entire data set, results in more accurate reconstructions of hyperspectral pixels from random projections.
\end{abstract}

Index Terms-Dimensionality reduction, Gaussian mixture model (GMM), hyperspectral data, random projection, support vector machine (SVM).

\section{INTRODUCTION}

$\mathbf{H}$ YPERSPECTRAL images (HSIs) acquired by remotesensing systems (e.g., spaceborne or airborne sensors) typically record reflectance values over a wide range of the electromagnetic spectrum for each pixel in the image. Although this rich spectral information potentially provides information useful to classification and analysis tasks, the high dimensionality of HSI poses challenges-including burdening transmission and storage systems as well as the reduced generalization capability of classifiers. Owing to the dense spectral sampling in HSI data cubes, not all features (or spectral bands) are expected to contribute useful information for the classification/analysis task at hand. Feature extraction is widely used as a prepro-

Manuscript received June 16, 2011; revised February 2, 2012; accepted June 4, 2012. Date of publication July 16, 2012; date of current version January 17, 2013. This work was supported in part by the National Science Foundation under Grant CCF-0915307 and in part by the University of Houston startup funds.

W. Li is with the Center for Spatial Technologies and Remote Sensing, University of California, Davis, CA 95616 USA (e-mail: liwei089@ ieee.org).

S. Prasad is with the Department of Electrical and Computer Engineering, University of Houston, Houston, TX 77204-4005 USA (e-mail: saurabh.prasad@ieee.org).

J. E. Fowler is with the Department of Electrical and Computer Engineering, Mississippi State University, Starkville, MS 39762 USA (e-mail: fowler@ece.msstate.edu).

Color versions of one or more of the figures in this paper are available online at http://ieeexplore.ieee.org.

Digital Object Identifier 10.1109/TGRS.2012.2204759 cessing technique to remove the redundancy in the original HSI spectral space and to potentially extract physically relevant information from it. Traditional feature-extraction methods include the discrete wavelet transform (DWT), independent component analysis [1], principal component analysis (PCA) [2], kernel PCA [3], locally linear embedding [4], and isometric feature mapping [5], among others.

In conventional HSI sensing systems, transformations such as a DWT or PCA are employed to reduce the spectral dimensionality at the sender side of a remote-sensing system. However, this paradigm has a disadvantage in that the computationally costly implementations of these transformations are required to reside on board the sender which is housed within the sensing modality. Typically, this sensor platform is a severely resource-constrained environment such as spaceborne remotesensing satellite. As an example, consider PCA, which is a data-dependent transform usually learned via a computationally expensive eigendecomposition - the computational cost of PCA is particularly significant when the spectral dimensionality of the HSI is high, hindering implementation on board the sensor platform.

As an alternative to such traditional transform-based sensing systems, there has been recent interest in accomplishing senderside dimensionality reduction via random projections. For example, compressed sensing (CS) (e.g., [6]-[8]) provides for the recovery of sparse signals from data-independent random projections. Alternatively, compressive-projection PCA (CPPCA) [9] also provides signal reconstruction from random projections. The CPPCA receiver not only recovers the coefficients associated with the PCA transformation but also approximates the PCA transform basis. Both CS and CPPCA avoid the computational burden of explicit sender-side dimensionality reduction and hence facilitate a lightweight sender. In [10], Fowler and Du presented a receiver-side hyperspectral reconstruction framework in which random projections are employed for the sensing/signal-acquisition process, while CS or CPPCA reconstruction takes place at the receiver-side base station.

Although classical analysis procedures may be applied after reconstruction via CS or CPPCA, some recent work has explored the efficacy of pattern classification, as well as target detection [11], [12], applied directly in the randomprojection domain without prior reconstruction. Additionally, [13] considers random projections for supervised learning with comparisons to PCA, while [14] describes the generalized maximum-likelihood-estimation (MLE) framework for compressive classification, offering theoretical bounds for classification accuracy. Furthermore, [15] combines random projection with the popular linear-discriminant-analysis (LDA) classifier 
in a randomly projected subspace, while [16] demonstrates that it is possible to train a soft-margin support-vector-machine (SVM) classifier in low-dimensional subspaces obtained by random projections. In [17], Dasgupta describes experiments on learning a Gaussian mixture model (GMM) in subspaces generated by random projections. Finally, [18] theoretically analyzes and applies a $K$-means clustering algorithm in randomly projected subspaces. The theoretical underpinning of all these approaches can be attributed to the Johnson-Lindenstrauss (JL) lemma [19]-[21] which asserts that if a low-dimensional subspace is selected randomly, interpoint distances of data points are approximately preserved.

Previous efforts at pattern classification and target detection within the domain of random projections (e.g., [11]-[18]) have focused exclusively on the process of conducting these analysis tasks in the random-projection subspace. This prior work has shown that, indeed, analysis such as classification can be performed surprisingly effectively in such lower dimensional spaces chosen at random. Consequently, the need to reconstruct the data set into its original dimensionality from the random projections is obviated, assuming that the application in question requires only classification or detection results from the data and not a full representation of the data set. However, this will not always be the case, as many situations will call for a reconstruction of the data set in its full dimensionality.

In this paper, we depart from this prior work in that we provide such full-dimensional reconstruction from the random projections. However, we do so in such a way so as to exploit the fact that we can effectively classify the data set into application-relevant regions prior to reconstruction and then drive the reconstruction so as to exploit the different properties of distinct regions. Specifically, a class-dependent reconstruction is proposed that effectively extends current reconstruction algorithms such as CPPCA [9].

In the proposed approach, after obtaining random projections of the hyperspectral image from the sender, each pixel is first labeled at the receiver as representing a distinct group/cluster/class in the scene (supervised grouping resulting from a supervised classifier, or unsupervised grouping resulting from an unsupervised clustering algorithm). After the grouping procedure, the CPPCA recovery algorithm is employed for the reconstruction of sets of pixels belonging to each cluster/class in this partition independently. The key motivation behind this strategy is that the traditional CPPCA algorithm recovers the primary eigenvectors representing the entire data set; however, when the data distribution in the original input space is distinctly multimodal, this approach may be suboptimal. On the other hand, the proposed class-dependent reconstruction recovers the primary eigenvectors for each distinct cluster separately, using them to recover pixels in that cluster independently of the others. In doing so, the overall receiver-side signal reconstruction from random projections is more accurate, since it is based on statistics which are representative of each of the dominant mixtures in the scene.

The remainder of this paper is organized as follows. In Section II, we start with a brief overview of the JL lemma and its ramifications for projection-domain classification. Then, in Section III, we review currently popular algorithms to re- construct HSI data from random projections, focusing on MTBayesian compressive sensing (multitask BCS) and CPPCA. In Section IV, we describe the proposed class-dependent CPPCA reconstruction strategies, following with Section V that presents an experimental analysis of their efficacy. Finally, some concluding remarks are made in Section VI.

As a final observation before proceeding, we note that the focus of this paper is exclusively on the issues of reconstruction from random projections of HSI. The ultimate feasibility of our of approach, however, is predicated on the successful design of hyperspectral sensors that are capable of performing random projection simultaneously with the signal-acquisition process within the sensing device. Although several sensing architectures for such have been proposed recently (e.g., [22] and [23]), these are, to our knowledge, too nascent to have been deployed on board any real air- or spaceborne platform as of yet.

\section{Classification in RANDom PRoJections}

Traditional supervised classification techniques, such as MLE and SVM classifiers [14], [16], [24], as well as unsupervised classification strategies, such as $K$-means and GMM clustering [17], [25], [26], are expected to work effectively in randomly projected subspaces due to the theoretical support of the JL lemma [19]-[21]. The JL lemma asserts that $L$ points in $\mathbb{R}^{N}$ can be projected into an $S$-dimensional subspace while approximately maintaining pairwise distances as long as $S$ is sufficiently large with respect to $L$. Specifically, for $\epsilon>0$ and every set $\mathcal{U}$ of $L$ points in $\mathbb{R}^{N}$, there exists a mapping $f: \mathbb{R}^{N} \rightarrow \mathbb{R}^{S}$ such that, for all $\mathbf{x}_{i}, \mathbf{x}_{j} \in \mathcal{U}$

$(1-\epsilon)\left\|\mathbf{x}_{i}-\mathbf{x}_{j}\right\|_{2}^{2} \leq\left\|f\left(\mathbf{x}_{i}\right)-f\left(\mathbf{x}_{j}\right)\right\|_{2}^{2} \leq(1+\epsilon)\left\|\mathbf{x}_{i}-\mathbf{x}_{j}\right\|_{2}^{2}$

as long as $S \geq O\left(\epsilon^{-2} \log L\right)$.

The JL lemma suggests that, for a sufficiently large $S$, the pairwise distances between points (i.e., the cluster structure) on a manifold are preserved with high probability under a random projection into an $S$-dimensional subspace. In other words, if a low-dimensional subspace is picked randomly, the projected clusters in the new space will capture the relevant information pertinent to classification present in their high-dimensional counterparts. This preservation of structure is critical to the performance of supervised/unsupervised classification techniques. In [13]-[18], it is observed that the overall accuracy of the classification/clustering of the data set in the randomly projected domain is equivalent (or at least close) to that in original data domain.

In our work, we employ the results of projection-domain classification or clustering to improve the reconstruction of the data set from those random projections. We first discuss the general problem of reconstruction from random projections in the next section before detailing our proposed class-dependent reconstruction in Section IV.

\section{Reconstruction From Random Projections}

Given a data set $\mathbf{X}=\left\{\mathbf{x}_{i}\right\}_{i=1}^{M}$ in $\mathbb{R}^{N}$ ( $N$-dimensional feature space), we assume that the signal-acquisition device at the 
sender applies an $N \times S$ orthonormal projection matrix $\mathbf{P}_{i}$ to vector $\mathbf{x}_{i}$ to obtain a random projection in $S$-dimensional subspace where $\mathbf{y}_{i}=\mathbf{P}_{i}^{\mathrm{T}} \mathbf{x}_{i}$ and $S \ll N$. We note that the ratio $S / N$ is referred to as the subrate hereafter. The problem at hand is then to recover $\mathbf{x}_{i}$ from $\mathbf{y}_{i}$ for all $i, 1 \leq i \leq M$.

\section{A. CS-Based Reconstruction}

CS literature [7], [8] tells us that if $\mathbf{x}_{i}$ can be sparsely represented with some $N \times N$ transform $\boldsymbol{\Psi}$, then we can recover $\mathbf{x}_{i}$ from the projections via a nonlinear reconstruction. The key to CS recovery is the production of a sparse set of significant transform coefficients, $\check{\mathbf{x}}_{i}=\boldsymbol{\Psi}^{-1} \mathbf{x}_{i}$, and the ideal recovery procedure searches for the $\check{\mathbf{x}}_{i}$ with the smallest $\ell_{0}$ norm consistent with $\mathbf{y}_{i}$. However, since this $\ell_{0}$ optimization is NP-hard, several alternative solutions have been proposed. Perhaps the most prominent of these is basis pursuit [27] which applies a convex relaxation to the $\ell_{0}$ problem resulting in an $\ell_{1}$ optimization

$$
\check{\mathbf{x}}_{i}=\arg \min _{\check{\mathbf{x}}}\|\check{\mathbf{x}}\|_{1}, \quad \text { such that } \mathbf{y}_{i}=\mathbf{P}_{i}^{\mathrm{T}} \mathbf{\Psi} \check{\mathbf{x}} .
$$

When the CS problem as expressed by (2) is treated from a Bayesian perspective, $\check{\mathbf{x}}_{i}$ in the basis $\Psi$ is expected to be sparse. BCS [28] formulates an a posteriori probability distribution for $\check{\mathbf{x}}_{i}$ and then provides confidence intervals in the approximation of $\mathbf{x}_{i}$ which can be used to guide the optimal design of additional CS measurements with the goal of reducing the uncertainty in $\mathbf{x}_{i}$. With noisy measurements (i.e., zero-mean Gaussian noise), BCS has the Gaussian likelihood model

$$
p\left(\mathbf{y}_{i} \mid \mathbf{x}_{i}, \sigma^{2}\right)=\left(2 \pi \sigma^{2}\right)^{-M / 2} \exp \left(-\frac{1}{2 \sigma^{2}}\left\|\mathbf{y}_{i}-\mathbf{P}_{i}^{\mathrm{T}} \mathbf{x}_{i}\right\|^{2}\right)
$$

where the matrix $\mathbf{P}_{i}$ is known and $\sigma^{2}$ is the noise variance. BCS chooses a Laplace density as the prior, and it is straightforward to see that the method seeks a full posterior density function for $\mathbf{x}_{i}$ and $\sigma^{2}$.

Compared to other norm-based CS methods, BCS exhibits a better performance in reconstructing signals with noisy measurements and achieves a tighter approximation to the $\ell_{0}$ norm. In our application, we wish to simultaneously recover an $M$-vector hyperspectral data set $\mathbf{X}$ which possesses high intervector correlation, and hence, a "multitask" extension of BCS, called MT-BCS [29], is employed.

\section{B. $C P P C A$}

Consider $M$ zero-mean vectors $\mathbf{X}=\left\{\mathbf{x}_{i}\right\}_{i=1}^{M}$ and assume that the same projection matrix is used for each $\mathbf{x}_{i}$, i.e., $\mathbf{P}_{i}=\mathbf{P}$ so that

$$
\mathbf{Y}=\mathbf{P}^{\mathrm{T}} \mathbf{X}
$$

In this case, the covariance matrix is $\boldsymbol{\Sigma}=\mathbf{X X}^{\mathrm{T}} / M$, and PCA seeks a linear transformation $\mathbf{W}$ of eigenvectors resulting from the eigendecomposition of $\Sigma$, i.e.,

$$
\boldsymbol{\Sigma}=\mathbf{W} \boldsymbol{\Lambda} \mathbf{W}^{\mathrm{T}}
$$

where $\mathbf{W}$ contains the $N$ unit eigenvectors of $\boldsymbol{\Sigma}$ columnwise. The PCA transform of $\mathbf{X}$ is then $\mathbf{X}=\mathbf{W}^{\mathrm{T}} \mathbf{X}$. On the other hand, the projected vectors have covariance

$$
\widetilde{\boldsymbol{\Sigma}}=\mathbf{Y} \mathbf{Y}^{\mathrm{T}} / M=\mathbf{P}^{\mathrm{T}} \mathbf{X} \mathbf{X}^{\mathrm{T}} \mathbf{P} / M=\mathbf{P}^{\mathrm{T}} \boldsymbol{\Sigma} \mathbf{P} .
$$

In our problem, the receiver obtains $\mathbf{Y}$ from the sender and can thus calculate $\widetilde{\boldsymbol{\Sigma}}$. The goal is then to approximate $\mathbf{W}$ from $\widetilde{\boldsymbol{\Sigma}}$ without knowledge of $\boldsymbol{\Sigma}$.

The Rayleigh-Ritz theory [30] describes the relation between the eigenvectors $\boldsymbol{\Sigma}$ and those of $\widetilde{\boldsymbol{\Sigma}}$ as given by (6). Covariance matrix $\boldsymbol{\Sigma}$ has eigenvalues satisfying $\lambda_{1}(\boldsymbol{\Sigma}) \geq \ldots \geq$ $\lambda_{N}(\boldsymbol{\Sigma})$, and the corresponding unit eigenvectors are $\mathbf{w}_{n}$. On the other hand, the eigendecomposition of $\widetilde{\boldsymbol{\Sigma}}=\mathbf{P}^{\mathrm{T}} \boldsymbol{\Sigma} \mathbf{P}$ is $\widetilde{\boldsymbol{\Sigma}}=$ $\widetilde{\mathbf{U}} \widetilde{\mathbf{\Lambda}} \widetilde{\mathbf{U}}^{\mathrm{T}}$, wherein the columns of $\widetilde{\mathbf{U}}$ are the unit eigenvectors $\widetilde{\mathbf{u}}_{s}$, and the eigenvalues are $\lambda_{1}(\widetilde{\boldsymbol{\Sigma}}) \geq \ldots \geq \lambda_{S}(\widetilde{\boldsymbol{\Sigma}})$. The $S$ eigenvalues $\lambda_{s}(\widetilde{\boldsymbol{\Sigma}})$ are called Ritz values; additionally, there are $S$ vectors, known as Ritz vectors, defined as

$$
\mathbf{u}_{s}=\mathbf{P} \widetilde{\mathbf{u}}_{s}, \quad 1 \leq s \leq S .
$$

Finally, normalized projection $\mathbf{v}_{n}$ is defined as the orthogonal projection of $\mathbf{w}_{n}$ onto $\mathcal{P}$, normalized to unit length, i.e.,

$$
\mathbf{v}_{n}=\frac{\mathbf{P P}^{\mathrm{T}} \mathbf{w}_{n}}{\left\|\mathbf{P} \mathbf{P}^{\mathrm{T}} \mathbf{w}_{n}\right\|_{2}} .
$$

CPPCA is built upon the fact established in [9] that if the eigenvalues of $\boldsymbol{\Sigma}$ are sufficiently eccentric (meaning that the eigenvalues are widely separated from one another), then $\mathbf{u}_{s} \approx$ $\mathbf{v}_{s}$. Specifically, it was shown in [9] that if we let $\omega_{1}$ be the angle between the first Ritz vector and the first projected eigenvector, $\omega_{1}=\angle\left(\mathbf{u}_{1}, \mathbf{v}_{1}\right)$, then it is expected that

$$
\sin \omega_{1} \leq C \cdot \frac{\delta}{\delta_{1}}
$$

where $\delta / \delta_{1}$ is the gap bound defined in terms of the eigenvalue gaps, $\delta_{n}=\lambda_{n}(\boldsymbol{\Sigma})-\lambda_{N}(\boldsymbol{\Sigma})$, and $\delta=\sum_{n=2}^{N-1} \delta_{n}$ and $C$ is a constant that depends only on $N$ and $S$. Similar results were also shown for the subsequent Ritz vectors $\mathbf{u}_{s}, s \geq 2$.

For the recovery of a data set of randomly projected vectors, [9] presents a reconstruction algorithm which employs a projection-onto-convex-sets (POCS) [31] procedure to approximate the principal eigenvectors (PEs) $\mathbf{w}_{n}$. As discussed in [9], in order to use POCS, the data set is necessarily split into multiple partitions, and a different projection matrix is used for each partition. Specifically, the sender splits the $M$ vectors in $\mathbf{X}$ into $J$ partitions $\mathbf{X}^{(j)}, j=1, \ldots, J . \mathbf{X}^{(j)}$ contains $M_{j}$ vectors from $\mathbf{X}$ and is associated with its own randomly chosen projection matrix $\mathbf{P}^{(j)}$. Thus, (4) becomes

$$
\mathbf{Y}^{(j)}=\mathbf{P}^{(j)}{ }^{\mathrm{T}} \mathbf{X}^{(j)} .
$$

In [9], a modulo partition is used, such that $\mathbf{X}^{(j)}=\left\{\mathbf{x}_{i} \in\right.$ $\mathbf{X} \mid(i-1) \bmod J=j-1\}$. It was assumed that each $\mathbf{X}^{(j)}$ closely resembles the whole data set $\mathbf{X}$ statistically such that

$$
\boldsymbol{\Sigma}^{(j)}=\mathbf{X}^{(j)} \mathbf{X}^{(j)^{\mathrm{T}}} / M_{j} \approx \boldsymbol{\Sigma} .
$$


At the CPPCA receiver, covariance matrices $\widetilde{\boldsymbol{\Sigma}}^{(j)}=\mathbf{Y}^{(j)} \mathbf{Y}^{(j)^{\mathrm{T}}} / M_{j}$ and, subsequently, Ritz vectors $\mathbf{u}_{s}^{(j)}$ are calculated from $\mathbf{Y}^{(j)}$. Under the assumption that $\mathbf{u}_{s}^{(j)} \approx \mathbf{v}_{s}$, matrices

$$
\mathbf{Q}_{s}^{(j)}=\left[\mathbf{u}_{s}^{(j)} \operatorname{null}\left(\mathbf{P}^{(j)^{\mathrm{T}}}\right)\right]
$$

are formed, where null $(\cdot)$ denotes the null-space. Finally, a parallel implementation of POCS [31] then produces an approximation to the eigenvectors of $\mathbf{X}$ as

$$
\widehat{\mathbf{w}}_{s}^{(i)}=\frac{1}{J} \sum_{j=1}^{J} \mathbf{Q}_{s}^{(j)} \mathbf{Q}_{s}^{(j)^{\mathrm{T}}} \widehat{\mathbf{w}}_{s}^{(i-1)}
$$

for $s=1, \ldots, L$; here, $i$ is the iteration index, and the iterations are initialized with $\widehat{\mathbf{w}}_{s}^{(0)}=\sum_{j} \mathbf{u}_{s}^{(j)} / J$.

A total of $L$ eigenvectors are recovered using the POCS procedure of (13); these are assembled into an $N \times L$ matrix $\boldsymbol{\Psi}=\left[\begin{array}{lll}\widehat{\mathbf{w}}_{1} & \ldots & \widehat{\mathbf{w}}_{L}\end{array}\right]$. The CPPCA receiver then proceeds to recover the PCA coefficients by using a pseudoinverse

$$
\widetilde{\mathbf{X}}^{(j)}=\left(\mathbf{P}^{(j)^{\mathrm{T}}} \boldsymbol{\Psi}\right)^{\dagger} \mathbf{Y}^{(j)}
$$

The number of eigenvectors to recover is determined by the heuristic $L=\operatorname{round}(S / \log N)$ as proposed in [32]. As in [9], we use $J=20$ for the work here.

\section{Proposed Class-Dependent Reconstruction}

We now propose a class-dependent reconstruction strategy as an extension of the reconstruction algorithms previously introduced. In this approach, the pixels in the HSI data set are first labeled/classified into groups in the projected domain at the receiver-supervised grouping resulting from a conventional supervised classifier and unsupervised grouping resulting from an unsupervised clustering algorithm. After the grouping procedure, the reconstruction algorithm is employed for each class independently - it is expected that, in doing so, the overall receiver-side signal reconstruction from sender-side random projections will be more accurate. In the algorithm proposed in this paper, we focus on CPPCA instead of CS because, in previous work [9], [10], CPPCA has been found to outperform CS for both reconstruction performance and execution speed.

The CPPCA reconstruction algorithm is employed individually for every class/cluster based on the ground-cover classification/clustering obtained using sender-side projections of the HSI data. It is expected that the proposed class-dependent CPPCA strategy will be more accurate at the reconstruction task than conventional CPPCA. This can be attributed to multiple modes in the data distribution arising from the presence of multiple classes/objects in the image. Hence, this class-dependent CPPCA method will employ statistics pertinent to each class as opposed to the average statistics over all classes. Although the sender remains unchanged from that of the original CPPCA, at the receiver, we first adopt a supervised or unsupervised classification to partition the random projections into several class-based groups. The CPPCA reconstruction procedure is then employed separately in each group.

\section{A. Class-Dependent CPPCA}

In class-dependent CPPCA, the sender partitions the data set into $\mathbf{X}^{(j)}$ as before and produces $\mathbf{Y}^{(j)}$ with $\mathbf{P}^{(j)}$ as in (10). At the receiver, however, the $\mathbf{Y}^{(j)}$ are each further partitioned into $K$ groups based on the result of a projection-domain classification (either supervised or unsupervised-more on this hereinafter). That is, each $\mathbf{Y}^{(j)}$ is divided into $K$ groups, $\mathbf{Y}_{k}^{(j)}, k=1, \ldots, K$, based on this classification. Next, CPPCA reconstruction is employed independently on each of the class partitions. That is, for each $k$, we calculate $\widetilde{\boldsymbol{\Sigma}}_{k}^{(j)}=$ $\mathbf{Y}_{k}^{(j)} \mathbf{Y}_{k}^{(j)^{\mathrm{T}}} / M_{k, j}$ as well as Ritz vectors $\mathbf{u}_{k, s}$, where $M_{k, j}$ denotes the number of vectors in $\mathbf{Y}_{k}^{(j)}$. Then, (12) and (13) become

$$
\begin{aligned}
& \mathbf{Q}_{k, s}^{(j)}=\left[\mathbf{u}_{k, s}^{(j)} \operatorname{null}\left(\mathbf{P}^{(j)^{\mathrm{T}}}\right)\right] \\
& \widehat{\mathbf{w}}_{k, s}^{(i)}=\frac{1}{J} \sum_{j=1}^{J} \mathbf{Q}_{k, s}^{(j)} \mathbf{Q}_{k, s}^{(j)^{\mathrm{T}}} \widehat{\mathbf{w}}_{k, s}^{(i-1)}
\end{aligned}
$$

respectively. Finally, (14) becomes

$$
\widetilde{\mathbf{X}}_{k}^{(j)}=\left(\mathbf{P}^{(j)^{\mathrm{T}}} \boldsymbol{\Psi}_{k}\right)^{\dagger} \mathbf{Y}_{k}^{(j)}
$$

where the $L$ recovered eigenvectors for class $k$ form $\Psi_{k}=$ $\left[\begin{array}{lll}\widehat{\mathbf{w}}_{k, 1} & \ldots & \widehat{\mathbf{w}}_{k, L}\end{array}\right]$. The $\widehat{\mathbf{X}}_{k}^{(j)}$ are then merged into the final reconstructed data set $\widehat{\mathbf{X}}$.

The motivation for this splitting into the class-based partitions stems from the fact that PCA effectively makes an assumption of spatial stationarity and ergodicity such that the ensemble covariance of vector $\mathbf{x}_{i}, \boldsymbol{\Sigma}^{*}=E\left[\mathbf{x}_{i} \mathbf{x}_{i}^{\mathrm{T}}\right]$, is well approximated by the sample covariance over the data set as a whole, $\boldsymbol{\Sigma}=\mathbf{X X}^{\mathrm{T}} / M$. In this case, $\boldsymbol{\Sigma}^{*} \approx \boldsymbol{\Sigma}$, and (5) is close to the "true" eigendecomposition for $\mathbf{x}_{i}$, such that $\check{\mathbf{x}}_{i}=\mathbf{W}^{\mathrm{T}} \mathbf{x}_{i}$ provides a representation for $\mathbf{x}_{i}$ with most of its variance compacted into relatively few PCA coefficients in $\check{\mathbf{x}}_{i}$.

However, in real life, data sets rarely, if ever, exhibit such stationary and ergodic conditions. The farther $\boldsymbol{\Sigma}$ is from the true covariance $\Sigma^{*}$, the less compact and less efficient the PCA transform will be for $\mathbf{x}_{i}$. The class-dependent strategy we propose here is effectively intended to overcome the lack of stationarity and ergodicity across the data set as a whole. A single class-based partition $\mathbf{X}_{k}$ is likely to be closer to being stationary and ergodic than the data set as a whole. The covariance within a single class-based partition, $\boldsymbol{\Sigma}_{k}=\mathbf{X}_{k} \mathbf{X}_{k}^{\mathrm{T}} / M_{k}$, will then be closer to $E\left[\mathbf{x}_{i} \mathbf{x}_{i}^{\mathrm{T}}\right]$ for each vector in class $k$. The PCA transform arising from the eigendecomposition for class $k, \boldsymbol{\Sigma}_{k}=\mathbf{W}_{k} \boldsymbol{\Lambda}_{k} \mathbf{W}_{k}^{\mathrm{T}}$, thus provides a more efficient and compact PCA representation for each vector in $\mathbf{X}_{k}$. The proposed class-dependent CPPCA inherits these benefits of class-based partitioning since it effectively attempts to recover the PCA transform within each class partition independently.

As an example, let us consider the case of the first eigenvector, i.e., the PE that corresponds to the largest eigenvalue. The $\mathrm{PE}$ lies in the direction of maximum variance of the training data. The motivation for our work is that traditional CPPCA recovers the $\mathrm{PE}$ corresponding to the overall data set/image, 


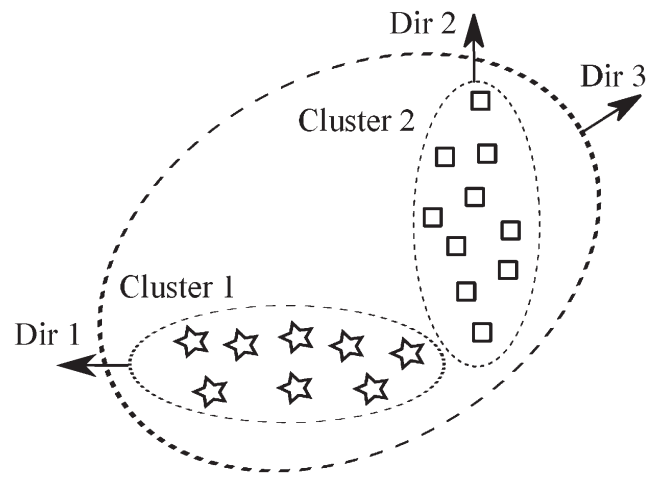

Fig. 1. Representative clustering and direction of PEs for synthetic data.

TABLE I

Gap Between the First and Second Eigenvalues, $\delta=\lambda_{1}-\lambda_{2}$

\begin{tabular}{c|c|c|c||c|}
\cline { 2 - 5 } \multicolumn{1}{c|}{ Dataset } & $\begin{array}{c}\text { first } \\
\text { cluster }\end{array}$ & $\begin{array}{c}\text { second } \\
\text { cluster }\end{array}$ & $\begin{array}{c}\text { third } \\
\text { cluster }\end{array}$ & $\begin{array}{c}\text { whole } \\
\text { dataset }\end{array}$ \\
\hline \hline Washington DC Mall & 13.98 & 14.81 & 13.28 & 8.40 \\
\hline University of Pavia & 20.35 & 9.76 & 7.62 & 5.28 \\
\hline Corn Field & 33.28 & 34.41 & 32.76 & 29.00 \\
\hline \hline
\end{tabular}

and we expect the reconstruction performance to be better when CPPCA is applied on each separate group instead of globally on the entire data set. Fig. 1 shows (with synthetic data) the benefit of clustering prior to CPPCA recovery. Suppose that the data set is composed of cluster 1 (marked by stars) and cluster 2 (marked by squares), which could possibly represent distributions of samples from two different materials. It is clear that the principal directions (denoted as Dir 1 and Dir 2 in Fig. 1) for cluster 1 and cluster 2 are significantly different. If CPPCA was to be applied on the entire data set globally, the estimated PE would be Dir 3, which is clearly not representative of the local statistics of either local cluster. Similar observations can be made for the other primary eigenvectors corresponding to the next largest eigenvalues as well.

As further evidence of the benefit of class-based partitioning, we consider the gap bound $\delta / \delta_{1}$ from (9). As mentioned previously, CPPCA reconstruction relies on the distribution of data being highly eccentric such that the primary eigenvalues are sufficiently distinct (i.e., widely separated in value) from one another. Table I presents the gap bound as calculated for several real hyperspectral data sets, comparing the gap bound for the eigenvalues as calculated from the covariance matrix for the entire data set against those calculated from three individual clusters produced in the image by the class-dependent strategy. From these results, it is clear that the class-dependent strategy significantly increases the eccentricity in the direction of the first eigenvector, providing a tighter bound in (9). This further corroborates our claim that a "local" cluster-dependent CPPCA will be more effective for signal reconstruction.

\section{B. Supervised Class-Dependent CPPCA}

We now introduce supervised class-dependent CPPCA for HSI reconstruction. By supervised, we mean that there are known classes in the HSI scene, and for the training process, labeled samples for those classes are available. As before, at the sender, the data set $\mathbf{X}$ is modulo partitioned and projected by measurement matrices $\mathbf{P}^{(j)}$, producing $\mathbf{Y}^{(j)}=\mathbf{P}^{(j)^{\mathrm{T}}} \mathbf{X}^{(j)}$. At the receiver, after obtaining the random projections $\mathbf{Y}^{(j)}$, we utilize a relatively small amount of $a$ priori training data (i.e., ground-truth) $\mathbf{X}_{\text {train }}$ to classify each pixel in each $\mathbf{Y}^{(j)}$ into one of $K$ classes. Thus, each $\mathbf{Y}^{(j)}$ is further partitioned into $K$ groups $\mathbf{Y}_{k}^{(j)}$. Finally, CPPCA reconstruction is employed independently on each of the class partitions.

Fig. 2 shows this supervised class-dependent CPPCA reconstruction strategy. We note that training data $\mathbf{X}_{\text {train }}$ is projected into the corresponding randomly projected subspace as $\mathbf{Y}_{\text {train }}^{(j)}$ via $\mathbf{P}^{(j)}$ before the classifier generates $\mathbf{Y}^{(j)}$. Additionally, the classification task can be undertaken by any supervised classifier, such as LDA-ML (LDA is commonly employed as a dimensionality-reduction preprocessing for the MLE classifier) or SVM, which classifies each $\mathbf{Y}^{(j)}$ into $K$ classes, assigning labels $C \in\left\{C_{1}, \ldots, C_{K}\right\}$ to the pixels.

\section{Unsupervised Class-Dependent CPPCA}

For situations when the a priori training information is unavailable, an unsupervised clustering strategy can be employed to partition the random projections $\mathbf{Y}^{(j)}$ for the proposed classdependent CPPCA approach. In fact, even without the training information, unsupervised class-dependent CPPCA may have a better reconstruction performance than its supervised counterpart. Consider the LDA-ML classifier for instance- the goal of LDA-ML is to reduce the classification error in the final classification map. Suppose we have a two-class problem, such as that shown in Fig. 3, wherein one of the classes has two distinct modes (marked by squares), while the other has one distinct mode (marked by stars). The class-label map of LDA-ML will not capture this structure and produce only two groups (the area of squares or stars). Nevertheless, unsupervised clustering may separate the data set into three groups which segment the square-labeled area into two clusters. According to our previous analysis, we see that unsupervised class-dependent CPPCA may be more relevant for signal reconstruction from random projections. We now consider two possible strategies for the incorporation of unsupervised clustering into CPPCA.

The most straightforward approach is to recover the entire data set $\mathbf{Y}$ first using CPPCA as shown in Fig. 4. Here, the unsupervised technique can be $K$-means, or a finite mixture model, which creates a clustering map wherein each pixel in the data set is assigned a label $C \in\left\{C_{1}, \ldots, C_{K}\right\}$, where $K$ is the number of clusters. Based on this map, we partition $\mathbf{Y}$ into $\mathbf{Y}_{k}, k=1, \ldots, K$. Similar to the procedure described in Section IV-B, CPPCA reconstruction is then employed independently on each class partition.

\section{Hybrid Unsupervised-Supervised Class-Dependent CPPCA With Random Partial Reconstruction}

We note that, although it would be possible to perform unsupervised clustering after the entire data is reconstructed using the CPPCA algorithm, we are particularly interested in the case wherein the clustering process is performed directly in the randomly projected domain. The potential challenge 


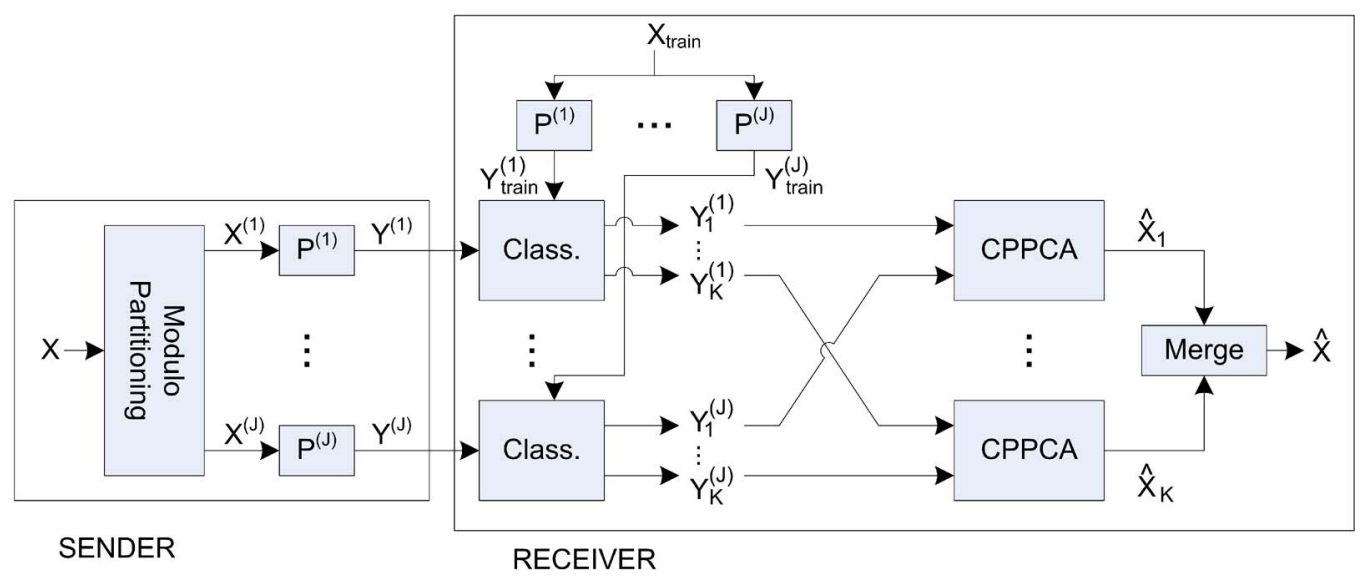

Fig. 2. Supervised class-dependent CPPCA.

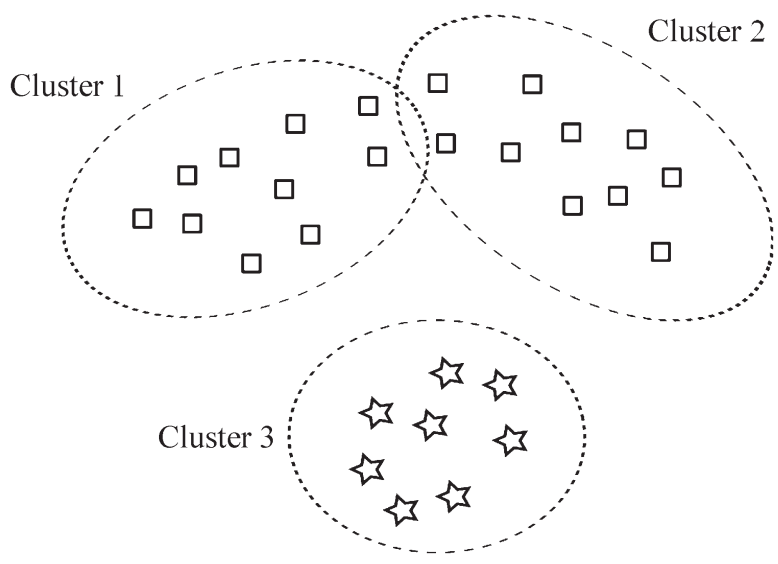

Fig. 3. Representative classification/clustering results for synthetic data.

with this is that projections $\mathbf{Y}^{(j)}$ are generated via separate measurement matrices $\mathbf{P}^{(j)}(j=1, \ldots, J)$. That is, when $j^{\prime} \neq j, \mathbf{Y}^{(j)}$ and $\mathbf{Y}^{\left(j^{\prime}\right)}$ are projected into different spaces wherein the structures are preserved differently, implying that we cannot use the clustering/classification methods directly in the domain of random projections. Furthermore, reconstituting results from these individual clustering operations into a unified partition $\left\{\mathbf{Y}_{k}\right\}_{k=1}^{K}$ for the entire HSI scene would be problematic.

However, it is possible to compromise and only partially reconstruct the data set from a small set of "exemplars" chosen randomly from the data set. Subsequently, an unsupervised clustering is used to partition these recovered exemplars into groups similar to Fig. 4. This can be viewed as producing "pseudo" a priori label information which can then be used to drive supervised class-dependent CPPCA reconstruction in a manner similar to Fig. 2. This resulting "hybrid" unsupervised-supervised reconstruction is shown in Fig. 5.

We note that, in Fig. 5, the reconstruction of the exemplars prior to clustering is performed using MT-BCS which can function well on the small number of exemplars (unlike CPPCA which requires a relatively large number of input vectors). The unsupervised clustering in Fig. 5 can be $K$-means or a finite mixture model.

\section{E. Hybrid Unsupervised-Supervised Class-Dependent CPPCA With Centroid Recovery}

As a variant of the hybrid unsupervised-supervised reconstruction discussed earlier, we can replace the random selection of exemplars with exemplars chosen to be close to the centroids of their associated clusters. That is, in Fig. 5, clustering, such as $K$-means, is performed separately in each subspace $\mathbf{Y}^{(j)}$. Then, instead of randomly selecting the exemplars from the $\mathbf{Y}^{(j)}$, we select exemplars close to the $K$ centroids of the $K$ clusters. These exemplars are then reconstructed using MT-BCS before driving unsupervised clustering and ultimately supervised classification as in Fig. 5. The key difference between this strategy and the one in Section IV-D is that the samples recovered by MT-BCS are representative of all clusters $\mathbf{Y}_{k}^{(j)}$ instead of being randomly chosen from the projections $\mathbf{Y}$.

\section{EXPERIMENTAL DATA AND RESUlts}

In this section, we describe the hyperspectral data used for various experiments in this work, as well as the procedure to tune the parameters of the proposed systems. We present experimental results with these class-dependent methods which demonstrate superior reconstruction performance as compared to conventional systems driven by global statistics. For simplicity, all projection matrices used are random orthonormal matrices generated by populating a matrix with independent Gaussian random variables followed by Gram-Schmidt orthogonalization.

\section{A. Hyperspectral Data}

The first experimental hyperspectral imagery employed to quantify the efficacy of the proposed approaches covers the Washington, DC, Mall area [33]. The image represents an urban scenario with $307 \times 1280$ pixels and 191 spectral bands in the 0.4-2.4- $\mu \mathrm{m}$ region of the visible and infrared spectra acquired by the Hyperspectral Digital Imagery Collection Experiment sensor with a spatial resolution of $1.5 \mathrm{~m}$. We used a size of $307 \times 307$ spatial cropping from the original image in our experiment. 


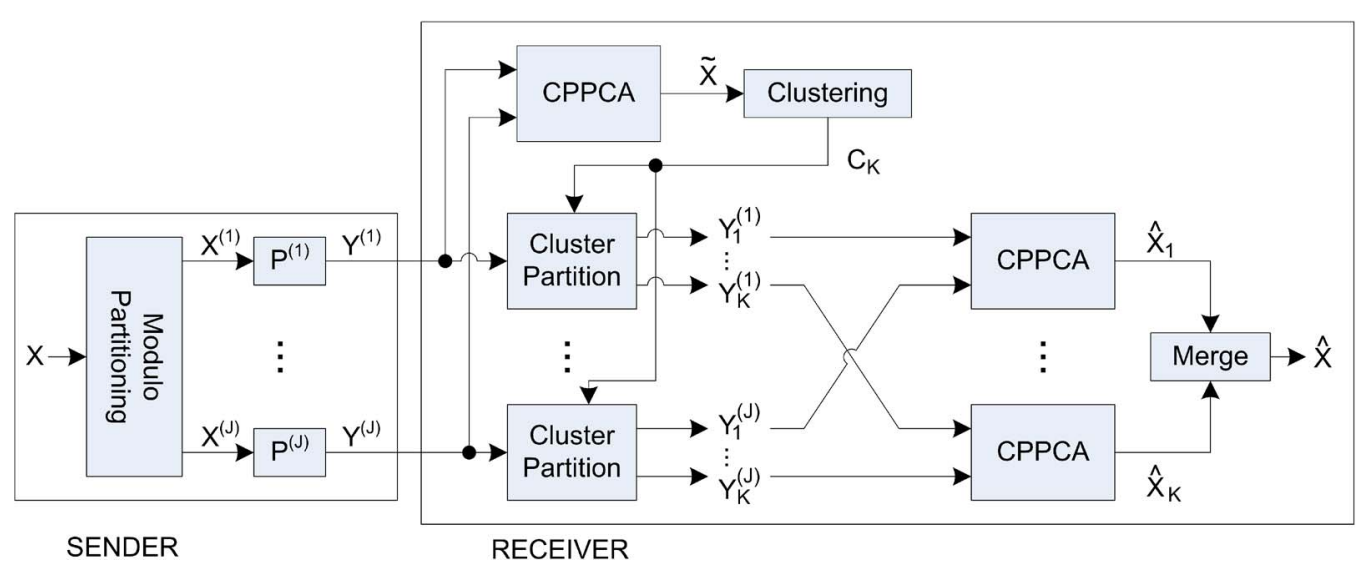

Fig. 4. Unsupervised class-dependent CPPCA.

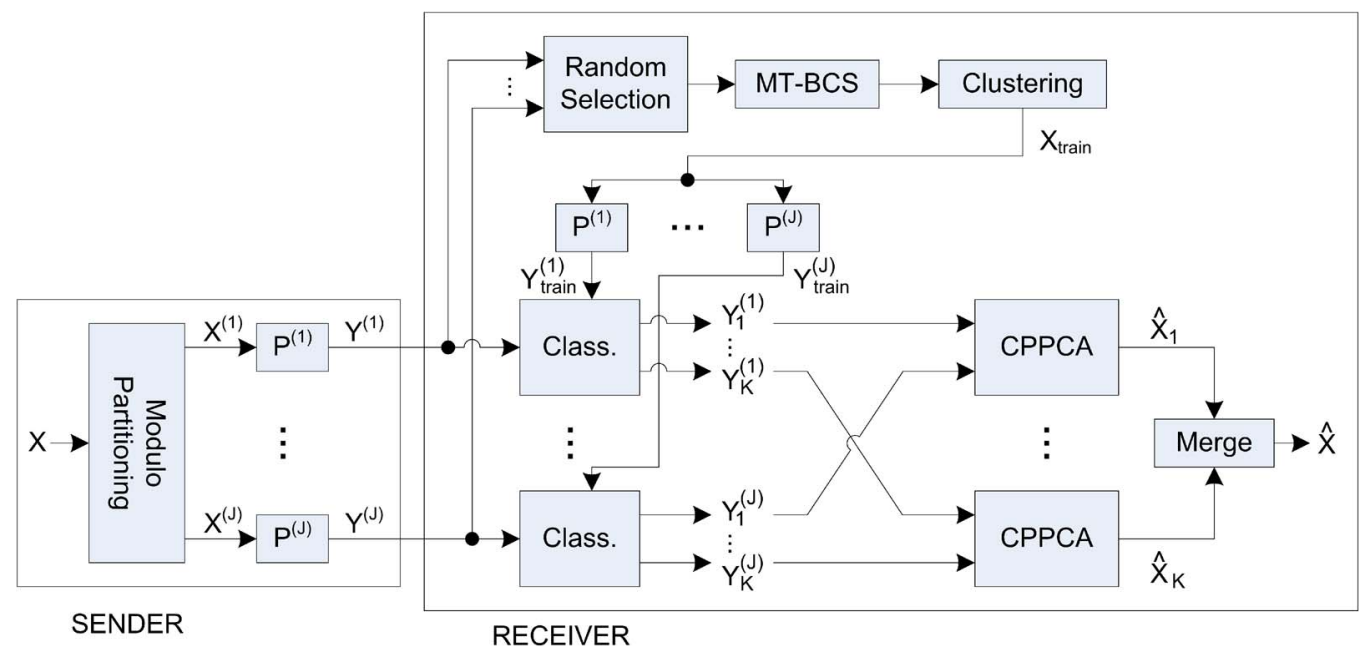

Fig. 5. Hybrid unsupervised-supervised class-dependent CPPCA (random partial reconstruction).

The second data set we employed was collected by the Reflective Optics System Imaging Spectrometer (ROSIS) sensor [34]. The flight over the city of Pavia, Italy, was in the framework of HySens project managed by the German Aerospace Agency (DLR). The images have 115 spectral bands with a spectral coverage from the $0.43-0.86-\mu \mathrm{m}$ region and a spatial resolution of $1.3 \mathrm{~m}$. The scene of the University of Pavia area, which has 103 spectral bands with a spatial size of $610 \times$ 340 pixels, is used in our experiment.

The third hyperspectral data set we used covers an agricultural (experimental corn crop) field in Brooksville, Mississippi. It was collected using ProSpecTIR visible near-infrared (VNIR) sensor on June 6, 2008. The images have 128 spectral bands with a spectral coverage of the $0.4-0.994-\mu \mathrm{m}$ region and a spatial resolution of $1.0 \mathrm{~m}$. This data set represents the corn crop under varying degrees of chemical-induced stress-each degree of stress in the crop is a unique class in this data set. The scene has a spatial size of $283 \times 213$ pixels.

\section{B. Parameter Tuning}

1) Classification Performance: Fig. 6 shows the classification performance for the Washington, DC, Mall data in the domain of random projections, which is compared with the

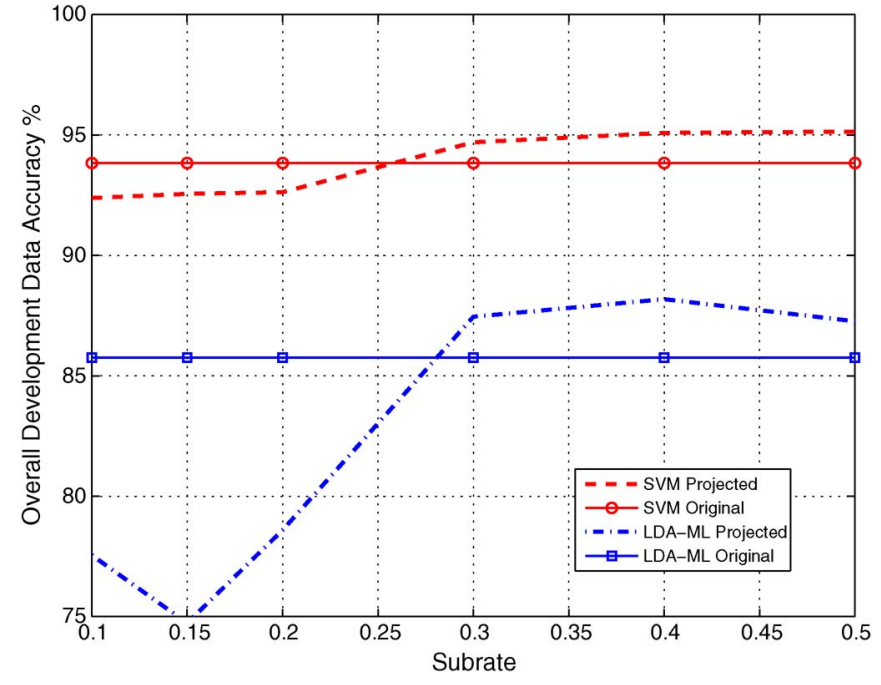

Fig. 6. Overall classification accuracy versus varying subrate in the randomly projected subspace for the Washington, DC, Mall data.

performance in the original data space. In this experiment, the training data are from five classes (roofs, streets/paths, grass, trees, and water). Approximately 2000 samples (383, 402, 405, 405, and 404 for each class, respectively) are employed for training, and approximately 5800 samples (1217, 1244, 1272, 
815 , and 1255 for each class, respectively) are employed for testing.

In Fig. 6, "SVM Projected" and "LDA-ML Projected" indicate that SVM and LDA-ML classifiers are employed to test the classification performance in the projected domain. "SVM Original" and "LDA-ML Original" indicate that SVM and LDA-ML classifiers are employed in the original data domain. It is clear from Fig. 6 that performing reliable ground-cover classification in the randomly projected subspace is indeed possible. In fact, we can also observe that the classification accuracies in the random-subspace domain are slightly higher compared to those of the same classifier employed in the original data domain when the subrate is larger than 0.3 (results for the two other data sets are similar). In the experiment, the dimensionality of projected space after an LDA transformation is upper bounded to $K-1$ by design ( $K$ is the number of classes in the classification task). For SVMs, data are normalized to have a range of $[0,1]$, and an RBF kernel with $\sigma=0.1$ was employed (all values were determined by tuning the system with development data over a range of values for this free parameter).

2) Number of Clusters: Both $K$-means and GMM clustering need knowledge of the number of clusters to be estimated in advance. However, in most practical situations, the value for the number of clusters $(K)$ is unknown. Choosing the number of clusters is traditionally always a problem for unsupervised clustering algorithms. Here, we adopt the Bayesian information criterion (BIC) [35] to determine the "optimal" value of $K$, which can also be found in [26] and [36], i.e.,

$$
B I C=-2 \mathcal{L}_{\max }(\Theta, \mathbf{X})+K \log M
$$

where $\mathcal{L}_{\max }(\Theta, \mathbf{X})$ is the maximum log-likelihood function for the model (parameter vector $\Theta$ ) and $M$ is the number of samples. A straightforward strategy for choosing $K$ is to set a maximum number of clusters for which BIC is estimated, and then, the optimal $K$ will be one which minimizes the value of BIC. For example, let the maximum number be set to ten. We calculate the BIC difference

$$
B I C_{\mathrm{diff}}=\frac{\|B I C(i)-B I C(i-1)\|_{1}}{\|B I C(i)\|_{1}} \leq \mathcal{T}_{\mathrm{BIC}}
$$

where $i=2,3, \ldots, 10$ and $\mathcal{T}_{\mathrm{BIC}}$ is a threshold. Fig. 7 shows the number of clusters versus the BIC difference values for the three data sets at a subrate of 0.3. The threshold is set to 0.02 , and the optimal number of clusters is the smallest value of $i$ that minimizes $B I C_{\text {diff }}$ under the threshold. As a result, in Fig. 7, we find the optimal number of clusters to be six for the Washington, DC, Mall data, seven for the University of Pavia data, and five for the corn-field data.

For GMM clustering, we adopt the diagonal-covariance model [36], meaning that all clusters have distinct but diagonalcovariance matrices. This is due to the dimensionality which causes the size of the resulting parameter space to be impractical if a full covariance matrix is employed.

Fig. 8 provides the classification/clustering maps for the University of Pavia data. In these maps, each color represents a unique class/cluster. In the proposed method, the class-

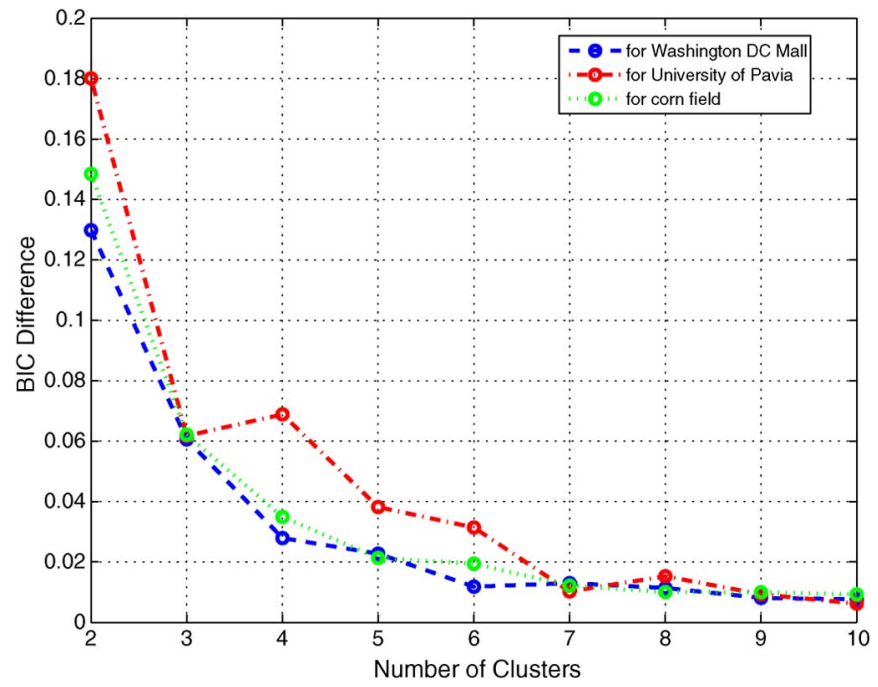

Fig. 7. Number of clusters versus BIC difference for the three data sets.

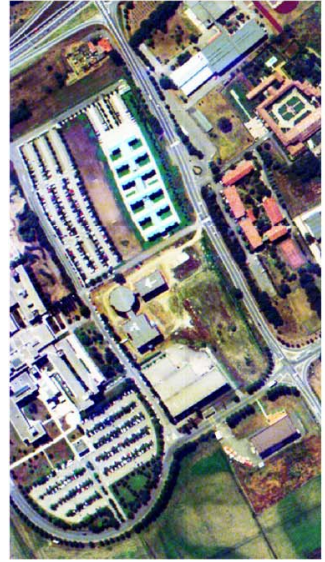

(a)

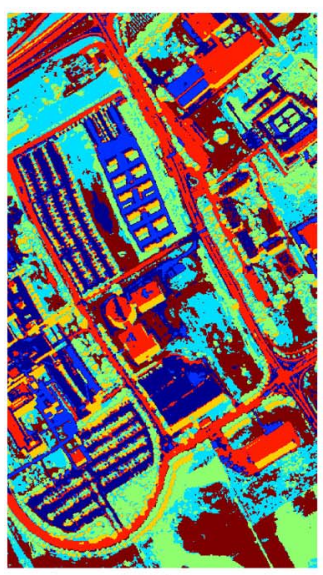

(c)

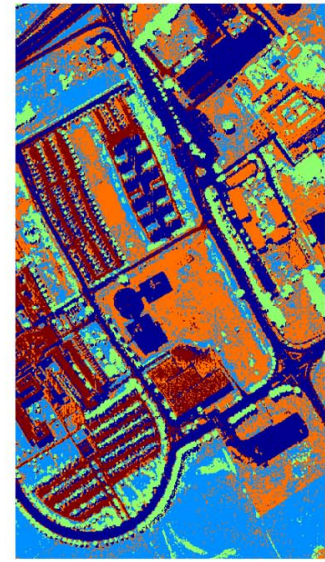

(b)

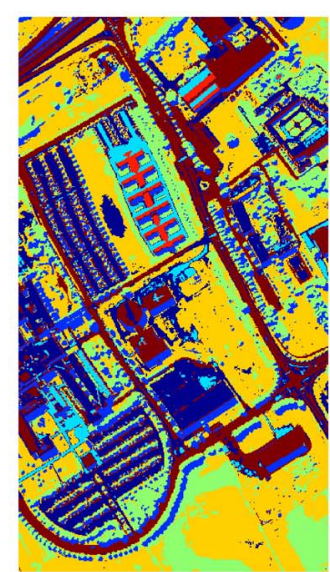

(d)
Fig. 8. (a) False color image of the University of Pavia data (generated using bands of 60,30, and 2), (b) classification map for the LDA-ML classifier for subrate 0.3 , (c) "CPPCA-KMEANS" with seven clusters, and (d) "CPPCA-Krandom" with seven clusters.

dependent CPPCA receiver performs reconstruction for each class/cluster independently. A similar observation was made for the other two data sets. 


\section{Experimental Results}

The efficacy of the proposed class-dependent CPPCA reconstruction strategy is measured by studying the signal-to-noise ratio (SNR) over a wide range of subrates [10]. In this paper, a vector-based SNR (measured in decibels) is employed

$$
S N R\left(\mathbf{x}_{i}, \mathbf{x}_{j}\right)=10 \log _{10} \frac{\operatorname{var}\left(\mathbf{x}_{i}\right)}{m s e\left(\mathbf{x}_{i}, \mathbf{x}_{j}\right)}
$$

where $\operatorname{var}\left(\mathbf{x}_{i}\right)$ is the variance of the data vector $\mathbf{x}_{i}$, the mean square error (mse) is

$$
\operatorname{mse}\left(\mathbf{x}_{i}, \mathbf{x}_{j}\right)=\frac{1}{N}\left\|\mathbf{x}_{i}-\mathbf{x}_{j}\right\|^{2}
$$

and $N$ is the dimensionality of feature space. We then average the vector-based SNR over all the vectors. ${ }^{1}$ Another popular evaluation method to measure the quality of reconstructed HSI is the average spectral angle [10], which is also commonly employed for detecting anomalous pixels in HSI. The spectral angle (measured in degrees) between the reconstructed vector and the corresponding original vector is defined as

$$
\xi_{m}=\angle\left(\mathbf{x}_{i}-\mathbf{x}_{j}\right)
$$

Then, $\xi_{m}$ is averaged over the entire data set, and the average spectral angle is $\xi=\operatorname{mean}\left(\xi_{m}\right)$.

The SNR and the average spectral angle for various reconstruction strategies are tabulated in Tables II and III. MT-BCS and CPPCA are adopted as two baseline algorithms whose efficacy is compared with that of the proposed methods

1) Referring to Section IV-B, the two supervised classdependent CPPCA algorithms are given the names CPPCA-LDA-ML (LDA-ML as the supervised classifier) and CPPCA-SVM (SVM as the supervised classifier).

2) Referring to Section IV-C, the two unsupervised classdependent CPPCA algorithms are given the names CPPCA-KMEANS ( $K$-means as the unsupervised clustering method) and CPPCA-GMM (GMM as the unsupervised clustering method).

3) Referring to Section IV-D, the hybrid unsupervisedsupervised class-dependent CPPCA algorithm with random partial reconstruction is given the name CPPCAK-random (after partial recovery by MT-BCS, $K$-means is employed as the clustering method to partition these reconstructions).

4) Referring to Section IV-E, another unsupervised classdependent CPPCA algorithm with partial reconstruction is given the name CPPCA-K-centroid (before the procedure of partial recovery, $K$-means is employed to segment each subspace $\mathbf{Y}^{(j)}$. Projected samples which are nearest to the centroid of each cluster $\mathbf{Y}_{k}^{(j)}$ are reconstructed by MT-BCS).

\footnotetext{
${ }^{1}$ In essence, we calculate the SNR for each pixel in the scenario and then average all the SNR values as the final SNR. The variance of the SNR values is approximately $4.92 \mathrm{~dB}$ when we test CPPCA for the Washington, DC, Mall data at subrate 0.3 in Table II. The variances (in decibels) are 4.01, 10.90, 9.95, and 10.34 for MT-BCS, CPPCA-LDA-ML, CPPCA-KMEANS, and CPPCAK-random, respectively.
}

\begin{tabular}{|c|c|c|c|c|}
\hline \multirow[b]{2}{*}{ Algorithms } & \multicolumn{4}{|c|}{ subrate } \\
\hline & 0.2 & 0.3 & 0.4 & 0.5 \\
\hline \multicolumn{5}{|c|}{ Washington DC Mall } \\
\hline MT-BCS & 20.23 & 24.92 & 29.21 & 32.44 \\
\hline CPPCA & 21.79 & 31.91 & 34.52 & 36.43 \\
\hline CPPCA-LDA-ML & 24.64 & 32.42 & 35.07 & 36.87 \\
\hline CPPCA-SVM & 27.95 & 33.25 & 35.87 & 37.33 \\
\hline CPPCA-KMEANS & 26.27 & 34.08 & 36.38 & 38.14 \\
\hline CPPCA-GMM & 29.47 & 34.49 & 36.70 & 38.25 \\
\hline CPPCA-K-random & 29.06 & 34.30 & 36.56 & 38.14 \\
\hline CPPCA-K-centroid & 29.02 & 34.35 & 36.51 & 38.07 \\
\hline \multicolumn{5}{|c|}{ University of Pavia } \\
\hline MT-BCS & 13.94 & 15.32 & 18.43 & 20.52 \\
\hline CPPCA & 14.29 & 16.77 & 20.62 & 22.99 \\
\hline CPPCA-LDA-ML & 15.38 & 19.19 & 21.73 & 23.68 \\
\hline CPPCA-SVM & 16.32 & 19.29 & 21.04 & 23.59 \\
\hline CPPCA-KMEANS & 17.53 & 20.28 & 22.19 & 23.86 \\
\hline CPPCA-GMM & 17.11 & 19.90 & 23.08 & 24.04 \\
\hline CPPCA-K-random & 16.61 & 19.38 & 22.61 & 24.23 \\
\hline CPPCA-K-centroid & 17.07 & 19.60 & 22.64 & 24.54 \\
\hline \multicolumn{5}{|c|}{ Corn Field } \\
\hline MT-BCS & 31.20 & 38.88 & 44.78 & 48.60 \\
\hline CPPCA & 36.92 & 42.40 & 46.12 & 47.92 \\
\hline CPPCA-LDA-ML & 38.16 & 43.33 & 47.11 & 49.12 \\
\hline CPPCA-SVM & 38.92 & 43.79 & 46.82 & 48.89 \\
\hline CPPCA-KMEANS & 40.67 & 44.91 & 48.23 & 50.45 \\
\hline CPPCA-GMM & 42.09 & 45.89 & 48.83 & 51.18 \\
\hline CPPCA-K-random & 40.84 & 45.26 & 48.37 & 50.62 \\
\hline CPPCA-K-centroid & 39.15 & 43.73 & 47.18 & 49.14 \\
\hline
\end{tabular}

TABLE II

Average SNR in Decibels Versus Varying Subrate

TABLE III

Average Spectral Angle in Degrees Versus Varying Subrate

\begin{tabular}{c|c|c|c|c|}
\cline { 2 - 5 } \multicolumn{4}{c|}{} & \multicolumn{4}{c|}{ Subrate } \\
\cline { 2 - 5 } Algorithms & 0.2 & 0.3 & 0.4 & 0.5 \\
\hline \hline Washington DC Mall \\
\hline CPPCA & 5.2682 & 1.3736 & 0.8024 & 0.6431 \\
\hline \hline CPPCA-LDA-ML & 4.3401 & 1.1980 & 0.7835 & 0.6373 \\
\hline CPPCA-SVM & 1.7804 & 0.9688 & 0.7077 & 0.6006 \\
\hline \hline CPPCA-KMEANS & 1.5910 & 0.8378 & 0.6651 & 0.5269 \\
\hline CPPCA-GMM & 1.3336 & 0.8010 & 0.6265 & 0.5244 \\
\hline \hline CPPCA-K-random & 1.5173 & 0.8363 & 0.6448 & 0.5269 \\
\hline CPPCA-K-centroid & 1.5487 & 0.8042 & 0.6569 & 0.5602 \\
\hline \hline \multicolumn{5}{|c|}{ University of Pavia } \\
\hline CPPCA & 3.7556 & 2.8786 & 1.8420 & 1.4087 \\
\hline \hline CPPCA-LDA-ML & 3.6055 & 2.1803 & 1.6016 & 1.2804 \\
\hline CPPCA-SVM & 3.5590 & 2.1689 & 1.6553 & 1.2942 \\
\hline \hline CPPCA-KMEANS & 2.8196 & 2.0491 & 1.5358 & 1.2682 \\
\hline CPPCA-GMM & 2.8258 & 2.0508 & 1.3907 & 1.2613 \\
\hline \hline CPPCA-K-random & 2.9025 & 2.1388 & 1.5183 & 1.2494 \\
\hline CPPCA-K-centroid & 2.8356 & 2.0545 & 1.3971 & 1.1487 \\
\hline \hline \multicolumn{5}{|c|}{ Corn Field } \\
\hline CPPCA & 0.5300 & 0.2737 & 0.1774 & 0.1443 \\
\hline \hline CPPCA-LDA-ML & 0.4437 & 0.2430 & 0.1569 & 0.1244 \\
\hline CPPCA-SVM & 0.4070 & 0.2279 & 0.1556 & 0.1251 \\
\hline \hline CPPCA-KMEANS & 0.3317 & 0.1958 & 0.1349 & 0.1046 \\
\hline CPPCA-GMM & 0.2872 & 0.1789 & 0.1270 & 0.0978 \\
\hline \hline CPPCA-K-random & 0.3339 & 0.1945 & 0.1350 & 0.1050 \\
\hline CPPCA-K-centroid & 0.4013 & 0.2226 & 0.1488 & 0.1225 \\
\hline \hline \multicolumn{5}{|c|}{} \\
\hline \hline
\end{tabular}

For CPPCA-LDA-ML and CPPCA-SVM, the ratios of the number of training labels to the total number of image pixels are $2.12 \%, 0.72 \%$, and $2.15 \%$ for these three HSI data sets, respectively. For CPPCA-K-random and CPPCA-K-centroid, 
we employ the SVM classifier for the pseudoclassification procedure since SVM works effectively when the training data set size is relatively small. Another reason we did not employ GMMs instead of $K$-means for these two strategies is the limited number of chosen projected samples. For CPPCA-Krandom, the number of samples recovered by MT-BCS is chosen as approximately 1500 for all the three data sets $(J=20$, so 75 samples from each subspace $\left.\mathbf{Y}^{(j)}\right)$. For CPPCA-K-centroid, ten samples nearest to the centroid are picked, which implies that $10 \times J \times K$ is the final number of samples recovered by MT-BCS. From Table II, the proposed supervised/unsupervised methods yield an average SNR substantially higher than conventional (i.e., global) MT-BCS and CPPCA over a range of practical subrates. For the urban Washington, DC, Mall data set, the proposed algorithms achieve a $2-8-\mathrm{dB}$ improvement in SNR. For the agriculture corn-field data set, the proposed methods provide a $1-5-\mathrm{dB}$ average gain. The relatively higher gain with the urban data sets can be attributed to the fact that the various classes in the scene are indeed very distinct, as opposed to the agriculture field data set for which the classes are representing the same species of a crop under different degrees of chemical-induced stress.

Insofar as computational complexity is concerned, we compare the execution speed of the proposed class-dependent CPPCA with that of the original CPPCA and MT-BCS for the Washington, DC, Mall data set at subrate 0.3. All implementations are run on a single desktop PC (quad core, $3.2 \mathrm{GHz}$ ) using Matlab R2009b-MT-BCS: 3660 s; CPPCA: 22 s; CPPCALDA-ML: 136 s; CPPCA-KMEANS: 47 s; CPPCA-K-random: $359 \mathrm{~s}$. We find that the proposed approaches generally run 10-70 times faster than MT-BCS but are still slower than CPPCA since they carry the additional burden of classification or unsupervised clustering.

\section{CONCLUSion}

In this paper, we have proposed the reconstruction of hyperspectral imagery in which pattern classification is combined with CPPCA reconstruction to recover data sets from random projections into lower dimensional subspaces. We have employed supervised/unsupervised classification to partition the image into distinct classes using sender-side projections. CPPCA is then applied to each class individually. In our experiments, we have analyzed and shown the efficacy of classification and clustering algorithms (i.e., Figs. 6 and 8) in the domain of random projections. We also have evaluated the reconstruction performance using SNR and average spectral angle. Experimental results have confirmed that the proposed methods consistently outperformed the conventional global reconstruction of the data set as a whole and are hence more suitable for HSI reconstruction from random projections.

$K$-means has been our primary choice for unsupervised clustering in this work because of its simplicity and fast execution. Although we have not included results with some other clustering techniques, such as spectral-clustering-based normalized cuts (Ncuts) [37], we have performed such experiments as well and found Ncut reconstruction to perform worse than $K$-means. We also have attempted to employ dimensionality-reduction techniques, such as PCA and locality preserving projections [38] as a preprocessing for clustering techniques ( $K$-means and finite mixture model) in the random-projected domain. However, we have found the performance to be no better than that without such preprocessing.

\section{ACKNOWLEDGMENT}

The authors would like to thank P. Gamba for the Reflective Optics System Imaging Spectrometer data and L. Mann Bruce for the ProSpecTIR VNIR data.

\section{REFERENCES}

[1] C. A. Shah, P. K. Varshney, and M. K. Arora, "ICA mixture model algorithm for unsupervised classification of remote sensing imagery," Int. J. Remote Sens., vol. 28, no. 8, pp. 1711-1731, Apr. 2007.

[2] S. Prasad and L. Mann Bruce, "Limitations of principal component analysis for hyperspectral target recognition," IEEE Geosci. Remote Sens. Lett., vol. 5, no. 4, pp. 625-629, Oct. 2008.

[3] H. Goldberg, H. Kwon, and N. M. Nasrabadi, "Kernel eigenspace separation transform for subspace anomaly detection in hyperspectral imagery," IEEE Geosci. Remote Sens. Lett., vol. 4, no. 4, pp. 581-585, Oct. 2007.

[4] S. T. Roweis and L. K. Saul, "Nonlinear dimensionality reduction by locally linear embedding," Science, vol. 290, no. 5500, pp. 2323-2326, Dec. 2000.

[5] A. Mohan, G. Sapiro, and E. Bosch, "Spatially coherent nonlinear dimensionality reduction and segmentation of hyperspectral images," IEEE Geosci. Remote Sens. Lett., vol. 4, no. 2, pp. 206-210, Apr. 2007.

[6] E. J. Candès and T. Tao, "Near-optimal signal recovery from random projections: Universal encoding strategies?" IEEE Trans. Inf. Theory, vol. 52, no. 12 , pp. 5406-5425, Dec. 2006.

[7] D. L. Donoho, "Compressed sensing," IEEE Trans. Inf. Theory, vol. 52, no. 4, pp. 1289-1306, Apr. 2006.

[8] E. J. Candès and M. B. Wakin, "An introduction to compressive sampling," IEEE Signal Process. Mag., vol. 25, no. 2, pp. 21-30, Mar. 2008.

[9] J. E. Fowler, "Compressive-projection principal component analysis," IEEE Trans. Image Process., vol. 18, no. 10, pp. 2230-2242, Oct. 2009.

[10] J. E. Fowler and Q. Du, "Reconstructions from compressive random projections of hyperspectral imagery," in Optical Remote Sensing: Advances in Signal Processing and Exploitation Techniques, S. Prasad, L. Mann Bruce, and J. Chanussot, Eds. Berlin, Germany: Springer-Verlag, 2011, ch. 3, pp. 31-48.

[11] M. A. Davenport, P. T. Boufounos, M. B. Wakin, and R. G. Baraniuk, "Signal processing with compressive measurements," IEEE J. Sel. Topics Signal Process., vol. 4, no. 2, pp. 445-460, Apr. 2010.

[12] K. Krishnamurthy, M. Raginsky, and R. Willett, "Hyperspectral target detection from incoherent projections: Nonequiprobable targets and inhomogeneous SNR," in Proc. Int. Conf. Image Process., Hong Kong, Sep. 2010, pp. 1357-1360.

[13] D. Fradkin and D. Madigan, "Experiments with random projection for machine learning," in Proc. ACM Conf. Knowl. Discovery Data Mining, Washington, DC, Aug. 2003, pp. 517-522.

[14] M. A. Davenport, M. F. Duarte, M. B. Wakin, J. N. Laska, D. Takhar, K. F. Kelly, and R. G. Baraniuk, "The smashed filter for compressive classification and target recognition," in Proc. Comput. Imag. V, vol. 6498, Proc. SPIE, San Jose, CA, Jan. 2007, p. $64980 \mathrm{H}$.

[15] R. J. Durrant and A. Kaban, "Compressive Fisher linear discriminant analysis: Classification of randomly projected data," in Proc. ACM Conf. Knowl. Discovery Data Mining, Washington, DC, Jul. 2010, pp. 1119-1128.

[16] R. Calderbank, S. Jafarpour, and R. Schapire, "Compressive learning: Universal sparse dimensionality in the measurement domain," Princeton Univ., Princeton, NJ, 2009, Tech. Rep.

[17] S. Dasgupta, "Experiments with random projections," in Proc. 16th Conf. Uncertainty Artif. Intell., Jun. 2000, pp. 143-151.

[18] C. Boutsidis, A. Zouzias, and P. Drineas, "Random projections for $k$-means clustering," in Advances in Neural Information Processing Systems, vol. 23, J. Lafferty, C. K. I. Williams, J. Shawe-Taylor, R. Zemel, and A. Culotta, Eds. Red Hook, NY: Curran, 2010, pp. 298-306.

[19] W. B. Johnson and J. Lindenstrauss, "Extensions of Lipschitz mappings into a Hilbert space," Contemporary Math., vol. 26, pp. 189-206, 1984. 
[20] S. Dasgupta and A. Gupta, "An elementary proof of a theorem of Johnson and Lindenstrauss," Random Struct. Algorithms, vol. 22, no. 1, pp. 60-65, Jan. 2003.

[21] D. Achlioptas, "Database-friendly random projections: JohnsonLindenstrauss with binary coins," J. Comput. Syst. Sci., vol. 66, no. 4, pp. 671-687, Jun. 2003.

[22] M. E. Gehm, R. John, D. J. Brady, R. M. Willett, and T. J. Schulz, "Singleshot compressive spectral imaging with a dual-disperser architecture," Opt. Exp., vol. 15, no. 21, pp. 14013-14 027, Oct. 2007.

[23] A. Wagadarikar, R. John, R. Willett, and D. Brady, "Single disperser design for coded aperture snapshot spectral imaging," Appl. Opt., vol. 47, no. 10, pp. B44-B51, Apr. 2008.

[24] W. Li, S. Prasad, J. E. Fowler, and L. Mann Bruce, "Locality preserving dimensionality reduction and classification for hyperspectral image analysis," IEEE Trans. Geosci. Remote Sens., vol. 50, no. 4, pp. 1185-1198, Apr. 2012.

[25] E. W. Forgy, "Cluster analysis of multivariate data: Efficiency versus interpretability of classification," Biometrics, vol. 21, no. 3, pp. 768-780, Sep. 1965.

[26] X. Z. Fern and C. E. Brodley, "Random projections for high dimensional data clustering: A clustering ensemble approach," in Proc. Int. Conf. Mach. Learn., Washington, DC, Aug. 2003, pp. 186-193.

[27] S. S. Chen, D. L. Donoho, and M. A. Saunders, "Atomic decomposition by basis pursuit," SIAM J. Sci. Comput., vol. 20, no. 1, pp. 33-61, Aug. 1998.

[28] S. Ji, Y. Xue, and L. Carin, "Bayesian compressive sensing," IEEE Trans. Signal Process., vol. 56, no. 6, pp. 2346-2356, Jun. 2008.

[29] S. Ji, D. Dunson, and L. Carin, "Multitask compressive sensing," IEEE Trans. Signal Process., vol. 57, no. 1, pp. 92-106, Jan. 2009.

[30] B. N. Parlett, The Symmetric Eigenvalue Problem. Philadelphia, PA: SIAM, 1998.

[31] P. L. Combettes, "The foundations of set theoretic estimation," Proc. IEEE, vol. 81, no. 2, pp. 182-208, Feb. 1993.

[32] W. Li and J. E. Fowler, "Decoder-side dimensionality determination for compressive-projection principal component analysis of hyperspectral data," in Proc. Int. Conf. Image Process., Brussels, Belgium, Sep. 2011, pp. 329-332.

[33] D. A. Landgrebe, "Hyperspectral image data analysis," IEEE Signal Process. Mag., vol. 19, no. 1, pp. 17-28, Jan. 2002.

[34] P. Gamba, "A collection of data for urban area characterization," in Proc. Int. Geosci. Remote Sens. Symp., Anchorage, AK, Sep. 2004, pp. 69-72.

[35] G. Schwarz, "Estimating the dimension of a model," Ann. Statist., vol. 6, no. 2, pp. 461-464, Mar. 1978.

[36] M. M. Dundar and D. Landgrebe, "A model-based mixture supervised classification approach in hyperspectral data analysis," IEEE Trans. Geosci. Remote Sens., vol. 40, no. 12, pp. 2692-2699, Dec. 2002.

[37] J. Shi and J. Malik, "Normalized cuts and image segmentation," IEEE Trans. Pattern Anal. Mach. Intell., vol. 22, no. 8, pp. 888-905, Aug. 2000.

[38] X. He and P. Niyogi, "Locality preserving projections," in Advances in Neural Information Processing System, S. Thrun, L. Saul, and B. Schölkopf, Eds. Cambridge, MA: MIT Press, 2004.

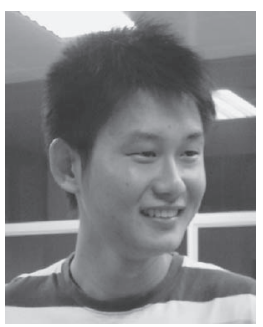

Wei Li (S'11) received the B.E. degree in electrical engineering from Xidian University, Xi' an, China, in 2007, the M.S. degree in electrical engineering from Sun Yat-Sen University, Guangzhou, China, in 2009, and the $\mathrm{Ph} . \mathrm{D}$. degree in electrical engineering from Mississippi State University, Starkville, in 2012.

$\mathrm{He}$ is currently a Postdoctoral Researcher with the Center for Spatial Technologies and Remote Sensing, University of California, Davis. His research interests include data compression, statistical pattern recognition, and hyperspectral image analysis.

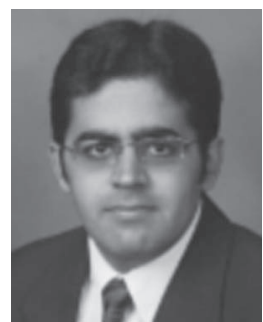

Saurabh Prasad (S'05-M'09) received the B.S. degree in electrical engineering from Jamia Millia Islamia, New Delhi, India, in 2003, the M.S. degree in electrical engineering from Old Dominion University, Norfolk, VA, in 2005, and the Ph.D. degree in electrical engineering from Mississippi State University, Starkville, in 2008.

$\mathrm{He}$ is an Assistant Professor with the Department Electrical and Computer Engineering and the Geosensing Systems Engineering Research Center, University of Houston, Houston, TX. He is an active Reviewer for the Elsevier Pattern Recognition Letters. He was the Lead Editor of the book entitled Optical Remote Sensing: Advances in Signal Processing and Exploitation Techniques (2011). His research interests include statistical pattern recognition, adaptive signal processing and kernel methods for medical imaging, and optical and synthetic aperture radar remote sensing. In particular, his current research work involves the use of information fusion techniques for designing robust statistical pattern classification algorithms for hyperspectral remote-sensing systems operating under low signal-to-noise ratio, mixed pixel, and small-training-sample-size conditions. He has been the lead author of several successful grant proposals to agencies such as National GeospatialIntelligence Agency, National Aeronautics and Space Administration, and the Department of Homeland Security and serves as the Principal Investigator and Technical Lead for these projects.

Dr. Prasad was a recipient of the Geosystems Research Institute's Graduate Research Assistant of the Year award in May 2007 and the Office-of-Research Outstanding Graduate Student Research Award in April 2008 at Mississippi State University, the Best Student Paper Award at IGARSS'2008 held in Boston, MA, in July 2008, and the State Pride Faculty Award at Mississippi State University for his academic and research contributions, in October 2010 $\mathrm{He}$ is an active Reviewer for the IEEE TRANSACTIONS ON GEOSCIENCE And Remote SENSING and the IEEE Geoscience And Remote SEnsing LETTERS.

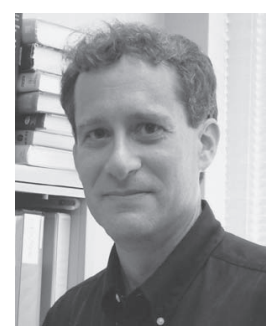

James E. Fowler (S'91-M'96-SM'02) received the B.S. degree in computer and information science engineering and the M.S. and Ph.D. degrees in electrical engineering from The Ohio State University, Columbus, in 1990, 1992, and 1996, respectively.

In 1995, he was an Intern Researcher with AT\&T Labs, Holmdel, NJ, and in 1997, he held a National Science Foundation-sponsored postdoctoral assignment with the Université de Nice, Sophia Antipolis, France. In 2004, he was a Visiting Professor with the Département Traitement du Signal et des Images, École Nationale Supérieure des Télécommunications, Paris, France. He is currently the Billie J. Ball Professor and Graduate Program Director of the Department of Electrical and Computer Engineering, Mississippi State University, Starkville, where he is also a Researcher with the Geosystems Research Institute. He is an Associate Editor for the EURASIP Journal on Image and Video Processing

Dr. Fowler is an Associate Editor for the IEEE TRANSACTIONS ON IMAGE PROCESSING; he formerly served as an Associate Editor for the IEEE TRANSactions on Multimedia and IEEE Signal Processing LetTers. He is the Chair of the Image, Video, and Multidimensional Signal Processing Technical Committee of the IEEE Signal Processing Society as well as the Publicity Chair of the program committee for the Data Compression Conference. 